J Child Adolesc Psychopharmacol Vol. 15(4):619-627 (2005)

ISSN: (print 1044-5463)(online 1557-8992)

doi:10.1089/cap.2005.15.619

This is a peer reviewed pre-print version of the following article: Zinc in Attention-Deficit/Hyperactivity Disorder, which has been published in final form at:

http://www.liebertpub.com

http://www.liebertpub.com/cap

http://www.liebertonline.com/doi/pdfplus/10.1089/cap.2005.15.619

(C) 2005 Mary Ann Liebert, Inc. Publishers

\title{
Zinc in Attention-Deficit/Hyperactivity Disorder
}

\section{Eugene Arnold, M.D., Department of Psychiatry, Ohio State University, Columbus, OH., Robert A. DiSilvestro, Ph.D., Department of Human Nutrition, Ohio State University, Columbus, $\mathrm{OH}$.}

\begin{abstract}
Objective:

The aim of this study was to review the published evidence for a role of zinc nutrition in attentiondeficit/hyperactivity disorder (ADHD).

Method:
\end{abstract}

A computer literature search was supplemented by the authors’ knowledge.

Results:

Numerous controlled studies report cross-sectional evidence of lower zinc tissue levels (serum, red cells, hair, urine, nails) in children who have ADHD, compared to normal controls and population norms. A few studies show correlations of zinc level with either clinical severity or a change thereof in response to stimulant or chemical challenge. Two placebo-controlled trials-one of zinc monotherapy, the other of zinc supplementation of methylphenidate-reported significant benefit. However, diagnostic procedures and sample representativeness were often not clear, and most such reports have come from countries and cultures with different diets and/or socioeconomic realities than are found in the United States (only one American sample in nine published reports). In particular, both positive clinical trials of zinc supplementation came from the Mid-East (Turkey and Iran), an area with suspected endemic zinc deficiency. The largest of these trials used zinc doses above the recommended upper tolerable limit and had a 2 in 3 dropout rate.

Conclusion:

It is not clear how well the accumulating evidence for a possible role of zinc in ADHD applies to middle-class American children. However, the evidence appears strong enough to warrant further controlled study in well-diagnosed samples representative of the socioeconomic spectrum. Hypothesistesting clinical trials are needed of this potential treatment that, if found effective, might become a relatively safe, cheap substitute for, or adjunct to, current treatments in some patients. At present, it should remain an investigational treatment.

\section{Introduction}

Attention deficit/hyperactivity disorder (ADHD) and its treatment is one of the most controversial areas in psychiatry. The disorder itself is well known to include inattention, distractibility, overactivity, and impulsivity excessive for developmental age, beginning by age 7, causing impairment in more than one setting, and not better explained by another disorder, according to Diagnostic and Statistical Manual of Mental Disorders, 4th edition (DSM-IV) criteria (APA1994). Debate begins with etiology and appropriate diagnosis and extends to 
outcome and treatment. Dozens of treatments have been advocated, from well-documented (such as U.S. Food and Drug Administration (FDA)- approved medication and behavioral treatment) at one pole to questionable or possibly dangerous at the other pole (Arnold 1999, 2004).

The more popular nonestablished etiological and treatment hypotheses include various nutritional foci. One of the more promising threads among these is a possible role of zinc in ADHD for some patients. This paper reviews the available published evidence relevant to such a possibility.

The authors' personal acquaintance with the zinc and ADHD literature was supplemented by a computer literature search. The peer-reviewed evidence falls into the categories of: Basic role in brain function, possible deficiency states, zinc measures in ADHD, association of ADHD symptoms with zinc levels, and two placebo-controlled trials of supplementation.

\section{Zinc and brain function}

Zinc is an important cofactor for metabolism relevant to neurotransmitters, prostaglandins, and melatonin and indirectly affects dopamine metabolism. It is necessary for 100 different metalloenzymes and metal-enzyme complexes (Toren et al. 1996). It contributes to the structure and function of the brain (Black 1998). For example, it is a coenzyme with delta-6desaturase, which is necessary for anabolism from dietary linolenic and linoleic acid of the longchain polyunsaturated fatty acids that make up neuronal membranes (e.g., Bettger et al. 1979). Much of the research has focused on fetal brain development (Wauben et al. 1999), but zinc also has a number of roles in the functioning of the postnatal developing, and developed, brain.

One biochemical and physiological role receiving increasing attention is zinc ion release during neuronal activity (Li et al, 2003). Approximately 15\% of the brain's zinc can be found in synaptic vesicles (Lopez-Garcia et al. 2001). Because these ions exhibit numerous effects on ligand-gated, voltage-dependent ion channels in vitro, zinc ions likely modulate synaptic transmission, though this is not directly confirmed (Li et al, 2003). Work in rats demonstrates that zinc is an important regulator of gamma-aminobutyric acid (GABA(A)) receptor function in the cortex (Schmid et al. 1999), restricts excitability of hippocampal glutamatergic neurons (Takeda et al 2003b), and is needed for brain tubulin growth and phosphorylation (Prasad 1993). Zinc deficiency in rats also affects protein synthesis (Prasad 1993), which is reflected in the brain by the altered expression of the P2X6 purinergic receptor (Chu et al 2003). Zinc can also affect the brain indirectly, because it is needed for cell membrane stabilization, indirect antioxidant functions, proper hormonal metabolism, and cellular energy release (Prasad 1993; Powell 2000). These functions have been shown to be affected by somewhat moderate zinc deficiencies in humans and/or experimental animals (Prasad 1993; Powell 2000; Devine et al. 1998; Licastro et al 1992). Zinc is necessary for the conversion of dietary pyridoxine (vitamin B6) to its active form, pyridoxal phosphate. In this form, vitamin B6 is necessary for the conversion of tryptophan to serotonin. Zinc is also necessary for the production and modulation of melatonin, which helps regulate dopamine function (Sandyk 1990; Chen et al. 1999), which is widely believed to be a key factor in attention-deficit/hyperactivity disorder (ADHD) and its treatment. In fact, Sandyk (1990) hypothesized that parasympathomimetic stimulants, at least amphetamine, work in ADHD partly through their effects on melatonin.

Although cellular mechanisms of zinc efflux and influx into brain cells are largely unknown, a zinc transporter has been identified in plasma membrane vesicles isolated from rat brain (Colvin 1998). Most of the zinc in the brain, except for the ionic zinc in synaptic vesicles, is bound tightly to proteins. As with other body tissues, much of the zinc in the brain is bound to metallothionein (Prasad 1993). Metallothionein I and II are structurally similar, low-weight 
proteins that seem important in drawing zinc into the cells and in free-radical scavenging (Prasad 1993; Disilvestro and Cousins 1984). Both the synthesis and degradation of metallothionein are very sensitive to zinc status (Prasad 1993, DiSilvestro and Carlson 1994; DiSilvestro and Cousins 1984; Thomas et al. 1992). The Brain contains not only metallothionein I and II, but also has a brain-specific metallothionein III (Zheng 1998). Although there is much more to be learned, it seems certain that brain function is influenced by this protein. For example, transgenic knock-out mice that lack the metallothionein III gene are highly sensitive to kainate-induced seizures (Zheng 1998; Takeda et al. 2003).

The relation to brain and other central nervous system (CNS) functions is also suggested by the effects of zinc deficiency. Some evidence from both animal and human studies suggests that zinc deficiency may affect cognitive development, though the mechanisms remain unclear (Bhatnagar and Taneja 2001). Postnatal moderate zinc deficiency in rats has demonstrated a number of brain effects, including impaired learned behavior and impaired cell maturation (Takeda et al. 2000; Yeiser et al. 2002). Chinese children with marginally deficient serum zinc levels improved neuropsychologic performance and growth upon zinc repletion, especially when accompanied by other micronutrients (Sandstead et al. 1998). There are also sensory and behavioral responses to dietary zinc alterations. For example, low food intake is a common finding in even marginally deficient rats (DiSilvestro et al. 1994). Low taste and smell sensitivity in zinc deficiency is well established in humans, though the degree of zinc deficiency needed to impair taste and smell is unclear (Alpers, 1994). Thus, zinc can influence brain and other CNS functions through a variety of mechanisms, and zinc deficiency can have detrimental effects on brain function.

Zinc deficiency has been identified in children from many parts of the world, especially in newly developing countries (Hambidge 2000; Prasad 1996). In the United States, severe zinc deficiency does not seem common among healthy children. However, a more subtle deficiency state may be. This state, sometimes called marginal zinc deficiency, has been identified by a number of studies, including studies of children from middle-income families (Hambidge 2000; Prasad 1996). Marginal zinc deficiency is also generally noted in nutritional texts (e.g., Wardlaw et al. 2004). Although its prevalence has not yet been studied, it is believed to be widespread. Diagnosis, so far, has been based on slow growth, low serum zinc values, and low hair-zinc values. All of these are reversed by increased zinc intake.

Frank zinc deficiency shows obvious physical signs of slowed growth, which might be considered analogous to scurvy from vitamin $\mathrm{C}$ deficiency. With increased knowledge, we now realize that somewhat higher levels of vitamin $\mathrm{C}$ may be optimal for more subtle functions, such as antioxidant effects. Analogously, marginal levels of zinc nutrition that do not result in frank physical signs might be suboptimal for brain physiology (e.g., Golub et al. 1996). A reverse analogy is lead toxicity, which formerly was diagnosed at levels above $50 \mathrm{mcg} / \mathrm{dL}$, then to 20 $\mathrm{mcg} / \mathrm{dL}$, but with increased knowledge of more subtle cognitive symptoms, the threshold was lowered to $10 \mathrm{mcg} / \mathrm{dL}$ by the Centers for Disease Control and Prevention (CDC) in 1991, and some experts now recommend single digits as the threshold (David et al. 1977; Kahn et al. 1995). A large-scale comparison of dietary data to recommended zinc intake suggests a pattern of marginally low intake in many people in the United States, including children (Briefel et al. 2000). Other, smaller dietary surveys also show such a pattern (Prasad 1993). This situation is likely not much improved by the intake of most multivitamin and mineral supplements, which typically use zinc oxide, which is not a well-absorbed form of zinc (Prasad et al. 1993; Anonymous 1994). 
Although moderately low zinc intake can be one factor in marginal zinc deficiency in U.S. children, other poorly identified factors could also play a role (Hambidge 2000). One such factor could be poor absorption. Acrodermatitis enteropathica is a recessive genetic disorder, in which zinc is poorly absorbed because of a defective transporter and which can be successfully treated by zinc supplementation (e.g., Perafan-Riveros 2002). It is not known whether the heterozygous condition might have mild impairment of zinc absorption, but the defective allele is believed rare and seems unlikely to contribute significantly to widespread marginal zinc deficiency or to a disorder as common as ADHD. A potentially more common cause of poor absorption could be a defect in the production of prostaglandins $E_{2}$ and $F_{2}$, which are necessary for zinc absorption (Song and Adham 1980). Yet another factor could be zinc-wasting metabolism, possibly precipitated by food additives or other ingested or environmental chemicals (Ward et al. 1990). Another possibility could be drug-induced effects on zinc; zinc intake could be just adequate enough for usual needs but not high enough for increased needs induced by drug action.

\section{State of art in measuring zinc deficiency}

One difficulty in pinpointing the scope of marginal zinc deficiency in the United States, as well as identifying negative effects of drugs on zinc status, has been the difficulty of assessing bodily stores of zinc. Plasma or serum zinc has been the traditional means of assessing zinc status (Sandstead and Alcock 1997; Thompson 1991; Prasad 1993). Values from serum and plasma are virtually identical (within the limits of lab error) and are considered interchangeable (e.g., English and Hambidge 1988). Low values can occur with severe or even marginal zinc deficiency and have diagnostic value. Nonetheless, this approach has two major limitations: (1) plasma zinc is not always sensitive to small changes in chronic zinc status (Thompson 1991; Bales et al. 1994); and (2) plasma/serum zinc values are affected by inflammation, other physiologic stress, and even by recent meals (Sandstead and Alcock 1997; Thompson 1991; Prasad 1993). Therefore, zinc investigators often measure zinc in several different types of specimen to get a more complete picture, such as serum, cells, hair, urine, and even nails. Rates of marginal deficiency, based on a single tissue assay, might be considered a lower-bound estimate.

Two other measures of zinc status have recently been found to be useful. One such measure is serum activity of zinc metalloenzyme plasma 5'-nucleotidase, which has the advantage of being sensitive to very small changes in zinc status. For example, Bales et al. (1994) have found that these activities respond to relatively small changes in zinc intake, over just 2 weeks or less, in human subjects, even in the absence of a change in plasma zinc. In addition, 5'-nucleotidase activities are low in mildly zinc-deficient rats (DiSilvestro RA, unpublished results), and extremely low in Type II diabetic subjects (Blostein-Fujii et al. 1997), a group prone to zinc deficiency (Moutschen et al. 1992). Activities rise in Type II diabetic women after zinc supplementation (Blostein- Fujii et al. 1997).

Another good measure for the assessment of marginal zinc status is erythrocyte metallothionein. Values have been depressed by experimental moderately low zinc diets in human volunteers (Grider et al. 1990; Thomas et al, 1992). A previous study showed that this parameter does not respond as quickly to mild zinc deficiency and repletion as does plasma 5'nucleotidase activities (Bales et al. 1994). However, this actually offers one advantage over the use of plasma 5'-nucleotidase activities alone to assess zinc status. Metallothionein values are especially useful for assessing long-term zinc status (Thompson 1991; Grider et al. 1990; 
Thomas et al. 1992). This is because values are not subject to short-term fluxes; a high zinc meal the day before blood sampling will not change the values.

\section{Zinc in ADHD}

Several data suggest that the marginal zinc deficiency described above may be more concentrated in the ADHD population—or, stated conversely, that the ADHD population may have a higher prevalence of marginal zinc deficiency. Both animal data (e.g., Halas and Sandstead 1975; Sandstead et al. 1977; Golub et al. 1996) and human findings suggest the involvement of zinc deficiency in ADHD symptoms. Studying moderately zinc-deprived monkeys, Golub et al. (1996) reported attentional impairment at levels that did not cause growth retardation. They concluded that activity and attention can be affected during the early stages of zinc deprivation before growth retardation results. Human zinc deficiency syndrome includes concentration impairment and jitters (Aggett and Harries 1979), and zinc deficiency can delay cognitive development (Black 1998).

In diagnosed ADHD, Kozielec et al. (1994) in Poland reported serum zinc significantly ( $p$ $<0.001$ ) deficient, compared to controls. Bekaroglu et al. (1996) in Turkey reported mean serum zinc of $60.6 \pm 9.9 \mathrm{mcg} / \mathrm{dL}$ in 33 boys and 15 girls with ADHD, compared to $105.8 \pm 13.2$ mcg/dL in healthy volunteers (30 boys and 15 girls). Toren et al. (1996), in Israel, reported significantly lower serum zinc levels and more variance in 39 boys and 4 girls 6-16 years of age with ADHD than in a control group of 28 age-matched healthy controls; 30\% of ADHD subjects were below the control range. In another Polish study, Starobrat-Hermelin (1998) found a high rate of magnesium, zinc, iron, copper, and calcium deficiencies in 116 children with ADHD on the basis of serum, red cell, and hair analyses. Hair zinc was lower in ADHD with comorbid oppositional-defiant or conduct disorder than in ADHD alone or with anxiety. Arnold et al. (1990) reported that 18 children with Diagnostic and Statistical Manual of Mental Disorders, 3rd edition, revised (DSMIII-R) ADHD had 30\% lower 24-hour urine zinc than 7 normal controls, suggesting either lower dietary intake or poorer absorption rather than zinc-wasting metabolism. In a companion paper (Arnold et al., 2005), we report on a negative correlation of serum zinc with parent- and teacher-rated inattentive symptoms ( $r=-45, p=0.004)$. Bekaroglu et al. (1996) concluded, “ . . . zinc deficiency may play a role in aetiopathogenesis of ADHD.” Sandyk (1990) suggests that the biochemical mechanism may be via effects on melatonin, which, he says, regulates dopamine function, which is known to be implicated in ADHD. Another possibility is that it may depend on the ionic zinc normally in the synaptic cleft (15\% of brain zinc). Rates of marginal deficiency, based on a single tissue assay, might be considered a lower-bound estimate; additional assays using plasma 5'- nucleotidase (Bales et al. 1994) and erythrocyte

metallothionein (Thompson 1991; Grider et al. 1990; Thomas et al. 1992) should discover more subtle marginal deficiency states.

\section{Interaction of zinc with synthetic chemicals}

There is a possibility of the exacerbation of marginal zinc deficiency by drug or other chemical interactions. Ward et al. (1990) in the United Kingdom found significantly lower zinc in 20 hyperactive boys, compared to 20 age-matched controls in urine $(p<0.001$, hair ( $p<$ $0.001)$, serum $(p<0.01)$, 24-hour urine $(p<0.01)$, and nails $(p<0.01)$. When 10 hyperactive boys and 10 age-matched controls from that sample were challenged with tartrazine-containing commercial beverages, serum and saliva zinc decreased while urine zinc increased in the hyperactive but not in the control boys, suggesting zinc wasting from the food-dye challenge. In a larger study, Ward (1997) found low zinc and iron in a sample of 486 hyperactive children, 
compared to 172 normal controls. When 47 of these subjects, with a parent-reported behavioral reaction to food dye, were challenged with $50 \mathrm{mg}$ of dye, their serum levels of zinc went down and urine levels went up, compared to age- and gender-matched normal controls. In both studies, the changes in zinc levels were associated with behavioral deterioration. Thus, the $5 \%-10 \%$ of children with ADHD who seem sensitive to dye or other food components may metabolically waste some of their zinc under chemical stress, exacerbating their already marginal status. These could constitute one subgroup with ADHD and marginal zinc.

\section{Possible effects on drug response}

Some evidence suggests that optimal stimulant response may depend on adequate zinc nutrition. Arnold et al. (1990) reported a significant correlation of baseline hair zinc with placebo-controlled d-amphetamine response on parent- and teacher-rated Conners hyperactivity index and hyperactivity factor ( $r=0.52$ to $0.61 ; p=0.02$ to 0.047 , two-tailed) in 18 boys $6-12$ years of age with ADHD. The same outcome variables also correlated nonsignificantly at $r=$ 0.30 to 0.45 with 24-hour urine zinc. In a reanalysis of the same data (Arnold et al. 2000), a pediatrician familiar with zinc assessments blindly categorized the 18 subjects as having good zinc nutrition ( $n=5)$, borderline zinc $(n=5)$, or mild or marginal zinc deficiency $(n=8)$ on the basis of hair, urine, and red-cell zinc levels. The deficiency was mild and not clinically evident and was picked up only by the tissue tests. The placebo-controlled amphetamine effects in the three zinc-nutrition groups on teacher ratings seemed associated linearly or asymptotically with the level of zinc nutrition. The placebo-controlled effect size (Cohen's d, difference between active-drug and placebo means divided by SD) of amphetamine on the Conners 10-item Hyperactivity Index was 1.37 in the presence of adequate zinc nutrition but only 0.55 in the presence of mild or marginal zinc deficiency, a difference of 0.82 (large) in the effect size with and without mild zinc deficiency. The "mildly zinc-deficient group” constituted $44 \%$ of that sample (8 of 18).

Akhondzadeh et al. (2004) examined zinc supplementation of methylphenidate response in Iranian children with ADHD. Forty-four children, 5-11 years of age, treated with methylphenidate $1 \mathrm{mg} / \mathrm{kg} /$ day in two divided doses, were randomly assigned to zinc sulfate 60 mg/day (22 children) versus placebo (22 children) for 6 weeks. On the DuPaul ADHD rating scale, those assigned to MPH plus supplemental zinc improved significantly more than those assigned to MPH plus placebo by parent $(p<0.05)$ and teacher $(p=0.04)$ ratings. Because of differences in endemic diet, it is not clear how applicable such Mid-Eastern findings are to American children with ADHD.

\section{Randomized clinical trial of zinc monotherapy}

Bilici et al. (2004) reported the only published trial of zinc supplementation alone as a treatment for ADHD, in a Turkish sample. It was flawed by a very high dropout rate (207 of 400) after randomization, as well as during a lead-in phase: Only 193 of the 618 consented eligible subjects completed the trial. Those randomized to zinc supplementation received $150 \mathrm{mg}$ of zinc sulfate/day for 12 weeks, a rather high dose, above the recommended upper tolerable limit. Instruments were either developed by the investigators (52-item clinician-rated ADHD scale on a 0-2 metric) or translated or adapted from English (Conners 28-item teacher and DuPaul 14-item parent rating). Thus, the outcome variables may not be comparable to other ADHD trials, thereby complicating an interpretation. The unusual quasiepidemiologic sample recruitment was both a strength and a weakness, raising questions about clinical representativeness, which was further compromized by excluding any child with comorbidity (even learning disorder and 
enuresis) or who was taking medicine. Nevertheless, after 12 weeks of treatment, the 46-item clinician-rated ADHD scale showed that the supplemented group ( $n=95$ completers, 202 total) improved by $25.4 \pm 9.7$ (an approximate $1 / 3$ decrease in score) compared to the placebo group ( $n$ = 98 completers, 198 total), which improved by only $12.7 \pm 5.4(p=0.002)$. The significant difference was a result of the hyperactivity, impulsivity, and impaired-socialization subscales, with no effect on the attention-deficit subscale. Those who were older and had higher body mass index and lower zinc and essential fatty-acid levels were most likely to benefit. Not all outcome measures were significant, and, unfortunately, it is not clear whether the cited data are for completers or totals. The report states, in one place, that the completers were used for the analysis, but in another place, it states that it was an intention-to-treat analysis with last observation carried forward. Thus, this study is promising but inconclusive.

\section{Cautions and limitations}

The literature reviewed in this paper do not prove that zinc deficiency is "the cause of ADHD” nor that zinc supplementation would be an effective treatment for ADHD. A number of alternative explanations for the findings could be postulated. Zinc levels could just be markers for some other cause of ADHD, including poor general nutrition, rather than having a direct causative role. For example, they could be markers of essential fatty-acid deficiency or imbalance because series 2 prostaglandins, derived from polyunsaturated fatty acids, are believed to be necessary for zinc absorption (Song and Adham 1980). They could even be markers of general malnutrition Wesnes et al (2003) reported that a whole-grain cereal breakfast prevented the deterioration of schoolchild attention and memory over the morning that was found in nobreakfast and glucose-drink conditions. Furthermore, zinc levels could be markers of a gene that results in deficient zinc absorption or metabolism and also affects attention but not necessarily through the zinc deficiency. Finally, they could be markers for inflammation-either infection, trauma, or allergy. Zinc is reduced in acute inflammation (Shenkin 1995). Hagerman and Falkenstein (1987) reported twice the rate of otitis media in hyperactive children compared to controls, suggesting either immune problems or greater exposure to infectious agents. Infectious toxins, allergies or sensitivities, and repeated trauma from the increased accident rate in ADHD could all contribute to an association of low zinc levels with ADHD without making low zinc the cause of ADHD. In other words, the low zinc could be an effect rather than a cause.

Yet another caution for American clinicians is that most of the published reports regarding zinc in ADHD involved samples other than American, raising questions about dietary differences and endemic regional deficiencies. Specifically, of nine published samples, two were Polish, two Turkish, one Iranian, one Israeli, two English, and only one American, with an additional American sample being published in a companion article (Arnold et al, 2005). This also raises questions about how similar the diagnostic procedures were to U.S. practice.

As a final caution, we should note that, although reasonably safe in RDA amounts, high doses can be toxic or at least cause side effects. At 50-150 mg/day, zinc can cause gastrointestinal upset and headache, and doses of $300 \mathrm{mg}$ /day can suppress immune function. Doses of $60 \mathrm{mg} /$ day may be enough to impair copper status in some adults (Prasad 1993; Wardlaw et al 2004); presumably, lower doses may cause this problem in children. The Turkish RCT (Bilici et al, 2004) used approximately $40 \mathrm{mg}$ of zinc per day (150 $\mathrm{mg} \mathrm{ZnSO}_{4}$ ), and it is possible that gastrointestinal side effects may have accounted for some of the high dropout rate (but the dropout rates were about the same for $\mathrm{ZnSO}_{4}$ and placebo).

\section{Conclusion}


The literature reviewed in this paper suggests the following: In addition to the animal data suggesting a link between zinc and ADHD-like symptoms, there are preliminary human data suggesting that: (1) many children with ADHD have lower-than-average zinc levels compared to both lab reference range and to normal control children; (2) zinc nutritional state may interact with ingested chemicals (drug or other), and (3) two Mid-Eastern trials showed greater improvement with zinc than with placebo.

These findings lead naturally to hypotheses that improving zinc nutritional status might improve the response to stimulants or might even have a beneficial effect independent of stimulants, or at least might lower the stimulant dose needed for benefit. These remain largely untested hypotheses at present (with the exception of the Turkish and Iranian trials) but deserve research attention because of the obvious potential public health importance. The data available at this time do not prove that low zinc causes ADHD nor make zinc supplementation an established treatment.

Possible foci for future research should include randomized clinical trials of zinc in welldiagnosed, broadly representative ADHD samples, both as monotherapy and as adjunct to open stimulant treatment; relative effect on attentional, hyperactive, and impulsive symptoms; effect on comorbid disorders, especially depression, for which there is also evidence of zinc involvement; relationship of zinc to other nutrients in ADHD; and effect on zinc tissue levels of food additives and drugs, including stimulant drugs.

\section{Acknowledgements}

The lead author (L.E.A.) has received research funding from Sigma Tau, Noven, Lilly, Novartis, Shire, and Targacept, receives speaker's honoraria from Novartis, Shire, and McNeil, and is a consultant for Sigma Tau, Dore, Noven, and Shire.

\section{References}

Agget PJ, Harries JT: Current status of zinc in health and disease states. Arc Dis Child 54: 909-917, 1979.

Akhondzadeh S, Mohammadi M-R, Khademi M: Zinc sulfate as an adjunct to methylphenidate for the treatment of attention-deficit/hyperactivity disorder in children: Adouble-blind, randomized trial. BMC Psychiatry 4(9), 2004.

Alpers DH: Zinc and deficiencies of taste and smell. JAMA 272:1233-1234, 1994.

APA (American Psychiatric Association Diagnostic \& Statistical Manual of Mental Disorders, 4 Edition (DSM-IV). Washington, DC. American Psychiatric Association, 1994.

APA (American Psychiatric Assoc.) Diagnostic \& Statistical Manual of Mental Disorders, 3rd Edition, Revised (DSH-III-R). Washinton, D.C. Amer Psychiatric Assoc, 1987.

Anonymous: Proceedings of the Albion International Nutritional Symposium., Clearfield, (UT), Albion Laboratories, 1994.

Arnold LE: Treatment alternatives for attention-deficit/hyperactivity disorder (ADHD). J Attent Disord 3:30-48, 1999.

Arnold LE: Methylphenidate versus Amphetamine: Comparative review. J Atten Disord 3(4): 200-211, 2000.

Arnold LE: Contemporary Diagnosis and Management of ADHD, 3rd edition. Newtown, (PA), Handbooks in Health Care, 2004.

Arnold LE, Hollway J, Bozzolo H, Cook A, DiSilvestro R, Bozzolo D, Crowl L, Ramadan Y, Williams C: Zinc correlates with parent-teacher-rated inattention in children with ADHD. J Child Adolesc Psychopharmacol 15(4):628-636, 2005.

Arnold LE, Pinkham SM, Votolato N: Does zinc moderate essential fatty acid and amphetamine treatment of attention-deficit/hyperactivity disorder? J Child and Adolesc Psychopharmacol 10(2):111-117, 2000.

Arnold LE, Votolato NA, Kleykamp D, Baker GB, Bornstein RA: Does hair zinc predict amphetamine improvement of ADHD/hyperactivity? Inter J Neurosci 50:103-107, 1990. 
Bales CW, DiSilvestro RA, Currie K, Plaisted CS, Joung H, Galanos AN, Lin P: Marginal zinc deficiency in older adults: Responsiveness of zinc status indicators. J Am Coll Nutr 13:455-462, 1994.

Bekaroglu M, Yakup A, Yusof G, Orhan D, Hilal M, Erol E, Caner K: Relationships between serum-free fatty acids and zinc and ADHD. J. Child Psychol Psychiatry 37:225B227, 1996.

Bettger WJ, Reeves PG, Moscatelli EA, Reynolds G, O’Dell BL: Interaction of zinc and essential fatty acids in the rat. J Nutr 109:480-488, 1979.

Bilici M, Yildirim F, Kandil S, Bekaroglu M, Yildirmis S, Deger O, Ulgen M, Yildiran A, Aksu H: Double-blind, placebo-controlled study of zinc sulfate in the treatment of attention-deficit/hyperactivity disorder. Prog Neuropsychopharmacol Biol Psychiatry 28:181-190, 2004.

Bhatnagar S: Taneja: Zinc and cognitive development. S Br J Nutr 85: S139-S145, 2001.

Black MM: Zinc deficiency and child development. Am J Clin Nutr 68 (Suppl 2): 464S-469S, 1998.

Blostein-Fujii A, DiSilvestro RA, Frid D, Katz C, Malarkey W: Short Term Zinc Supplementation in Type II Diabetic Women: Effects on Plasma 5'- Nucleotidase Activities, Insulin-Like Growth Factor Levels \& Lipoprotein Oxidation Rates in Vitro. Am J Clin Nutr 66:639-642, 1997.

Briefel RR, Bialostosky K, Kennedy-Stephenson J, McDowell MA, Ervin RB, Wright JD: Zinc intake in the U.S. population: Findings from the Third National Health and Nutrition Examination Survey, 1988-1994. J Nutr 130:1367S-1373S, 2000.

Chen MD, Lin PY, Sheu WH: Zinc coadministration attenuates melatonin's effect on nitric oxide production in mice. Bio Trace Elem Res 69(3):261-268, 1999.

Chu Y, Mouat MF, Coffield JA, Orlando R, Grider A: Expression of P2X6, a purinergic receptor subunit, is affected by dietary zinc deficiency in rat hippocampus. Bio Trace Elem Res 91: 77-87, 2003.

Colvin RA: Characterization of a plasma membrane zinc transporter in rat brain. Neurosci Lett 247:147-150, 1998.

Conners CK: Conners’ Rating Scales - Revised: Technical Manual. New York, MHS, 2001.

David OJ, Hoffman SP, Sverd J, Clark J: Lead and hyperactivity: Lead levels among hyperactive children. J Abnorm Child Psychol 5:405-416, 1977.

DiSilvestro RA, Carlson GP: Effects of mild zinc deficiency, plus or minus acute phase response, on CCl4 hepatotoxicity. Free Rad Biol Med 16:57-61, 1994.

DiSilvestro RA, Cousins RJ: Mediation of endotoxin-induced changes in zinc metabolism in rats. Am J Physiol 247:E436-E441, 1984.

Devine A, Rosen C, Mohan S, Baylink D, Prince RL: Effects of zinc and other nutritional factors on insulin-like growth factor I and insulin-like growth factor binding proteins in postmenopausal women. Am J Clin Nutr 68:200-206, 1998.

English JL, Hambidge KM: Plasma and serum zinc concentrations: effect of time between collection and separation. Clin Chim Acta 175:211-215, 1988.

Golub MS, Takeuchi PT, Keen CL, Hendrickx AG, Gershwin ME: Activity and attention in zinc-deprived adolescent monkeys. Am J Clin Nutr 64(6):908-915, 1996.

Grider A, Bailey LB, Cousins RJ: Erythrocyte metallothionein as an index of zinc status in humans. Proc Natl Acad Sci 87:1259-1262, 1990.

Hagerman RJ, Falkenstein AR: An association between recurrent otitis media in infancy and hyperactivity. Clin Pediatr 26:253-257, 1987.

Halas ES, Sandstead HH: Some effects of prenatal zinc deficiency on behavior of the adult rat. Pediatr Res, 9:94B97, 1975.

Hambidge M: Human zinc deficiency. J Nutr 130: 1344S-1349S, 2000.

Kahn CA, Kelly PC, Walker WO, Jr: Lead screening in children with ADHD and developmental delay. Clinl Pediatr 34:498-501, 1995.

Kozielec T, Starobrat-Hermelin B, Kotkowiak L: Deficiency of certain trace elements in children with hyperactivity (Polish). Psychiatr Pol 28(3): 345-353, 1994.

Li YV, Hough CJ, Sarvey JM: Do we need zinc to think? Sci STKE 182:19, 2003.

Licastro F, Mocchegiani E, Zannotti M, Arena G, Masi M, Fabris N: Zinc affects the metabolism of thyroid hormones in children with Down's syndrome: Normalization of thyroid-stimulating hormone and of reversal tri-iodothyronine plasmic levels by dietary zinc supplementation. Inter J Neurosci 65:259-268, 1992.

Lopez-Garcia C, Molowny A, Ponsoda X, Nacher J, Sancho-Bielsa F: Synaptic zinc in the central nervous system. Rev Neurol 33: 341-347, 2001. 
Moutschen MP, Scheen AJ, Lefebvre PJ: Impaired immune responses in diabetes mellitus: analysis of the factors and mechanisms involved. Relevance to the increased susceptibility of diabetic patients to specific infections. Diabete Metab 18:187-201, 1992.

Perafan-Riveros C, Franca LFS, Alves ACF, Sanches JA: Acrodermatitis enteropathica: Case report and review of the literature. Pediatric Dermatol 19:426-431, 2002.

Prasad AS: Zinc deficiency in women, infants, and children. J Am Coll Nutr 15:113-120, 1996.

Prasad AS: Biochemistry of Zinc, New York, Plenum Press, 1993.

Prasad AS, Beck FWJ, Nowak J: Comparison of absorption of five zinc preparations in humans using oral zinc tolerance test. J Trac Elem Exp Med 6:109-115, 1993.

Powell SR: The antioxidant properties of zinc. J Nutr 130:1447S-1454S, 2000.

Sandstead HH, Alcock NW: Zinc: An essential and unheralded nutrient. J Lab Clin Med 130(2):116-118, 1997.

Sandstead HH, Fosmire GJ, Halas ES, Jacob RA, Strobel DA, Marks EO: Zinc deficiency: Effects on brain and behavior of rats and Rhesus monkeys. Teratology 16:229B234, 1977.

Sandstead HH, Penland JG, Alcock NW, Dayal HH, Chen XC, Li JS, Zhao F, Yang JJ: Effects of repletion with zinc and other micronutrients on neuropsychologic performance and growth of Chinese children. Am J Clin Nutr 68:470S-475S, 1998.

Sandyk R: Zinc deficiency in attention-deficit/hyperactivity disorder. Inter J Neurosci 52:239B241, 1990.

Schmid G, Chittolini R, Raiteri L, Bonanno G: Differential effects of zinc on native GABA(A) receptor function in rat hippocampus and cerebellum. Neurochem Int 34:399-405.

Shenkin A: Trace elements and inflammatory response implications for nutritional support. Nutrition 11(1)(Suppl):100-105, 1995.

Song MK, Adham NF: Evidence for an important role of prostaglandins $E_{2}$ and $F_{2}$ in the regulation of zinc transport in the rat. J Nutr 109:2152-2159, 1980.

Starobrat-Hermelin B: The effect of deficiency of selected bioelements on hyperactivity in children with certain specified mental disorders. Ann Acad Med Stetin 44:297-314, 1998.

Takeda A, Hirate M, Tamano H, Nisibaba D, Oku N: Susceptibility to kainate-induced seizures under dietary zinc deficiency. J Neurochem 85:1575-1580, 2003a.

Takeda A, Hirate M, Tamano H, Oku N: Release of glutamate and GABA in the hippocampus under zinc deficiency. J Neurosci Res 72:537-542, 2003b.

Takeda A, Takefuta S, Okada S, Oku N: Relationship between brain zinc and transient learning impairment of adult rats fed zinc-deficient diet. Brain Res 859:352-357, 2000.

Thomas EA, Bailey LB, Kauwell GB, Lee DY, Cousins RJ: Erythrocyte metallothionein response to dietary zinc in humans. J Nutr 122:2408-2414, 1992.

Thompson RPH: Assessment of zinc status. Proc Nutr Soc 50:19-28, 1991.

Toren P, Sofia E, Sela BA, Wolmer L, Weitz R, Dov I, Koren S, Reiss A, Weizman R, Laor N: Zinc deficiency in ADHD. Biol Psychiatry 40:1308B-1310B, 1996.

Ward NI: Assessment of chemical factors in relation to child hyperactivity. J Nutr Environ Med (Abingdon) 7(4):333-342, 1997.

Ward NI, Soulsbury KA, Zettel VH, Colquhoun ID, Bunday S, Barnes B: The influence of the chemical additive tartrazine on the zinc status of hyperactive children: A double-blind, placebo-controlled study. J Nutr Med 1(1):51-58, 1990.

Wardlaw GM, Hampl JS, DiSilvestro RA: Perspectives in Nutrition, 6th ed. New York, McGraw Hill, 2004.

Wauben IP, Xing HC, Wainwright PE: Neonatal dietary zinc deficiency in artificially reared rat pups retards behavioral development and interacts with essential fatty acid deficiency to alter liver and brain fatty acid composition. J Nutr 129:1773-1781, 1999.

Wesnes KA, Pincock C, Richardson D, Helm G, Hails S: Breakfast reduces declines in attention and memory over the morning in schoolchildren. Appetite 41:329-331, 2003.

Yeiser EC, Vanlandingham JW, Levenson CW: Moderate zinc deficiency increases cell death after brain injury in the rat. Nutr Neurosci 5:345-352, 2002.

Zheng J: Research progress in brain-specific metallothionein-III. Prog Physiol 29:325-330, 1998. 